\title{
Camera Perspectives Influence of Apparel E-commerce Images on Visual Attention and Consumer Purchase Interest: Evidence from Eye Tracking
}

\author{
Y.T. HUANG \\ Department of Commercial Design, Chung Yuan Christian University, Taiwan \\ H.F. HO \\ Department of Electrical Engineering, National Taiwan Normal University, Taiwan
}

\begin{abstract}
This study implements the eye-tracking method to analyze participants' eye movement data to stimuli in order to assess images from different camera perspectives of apparel clothing on online shopping websites. The results show consumer preferences for camera perspectives on apparel e-commerce: images with models displaying the item are better than enlarged images of the single item; images with scenario backgrounds receive more visual attention than images with the background removed, images with scenario backgrounds also provide customers greater mental stimulation for use of the product; although the participants answered that they prefer models with their faces visible when displaying the item, however through the eye-tracking experiment, results show that images where model's faces are not visible receive greater visual attention.
\end{abstract}

KEYWORD: camera perspective; e-commerce; eye movement; visual attention; visual behavior.

\section{INTRODUCTION}

As information technology and the global economic structure changes, a new type of consumer group have evolved since the year 2000, a group that is lacking in time and focus[1]. This type of new consumer group is dependent upon the everdeveloping e-commerce system to conduct shopping with rapid speed and decision; in response, traditional marketing channels have set up virtual online platforms to expand their business. However, as marketing becomes more challenging and platform technology innovates, how online brands integrate visual design and layout - such as graphic images and text messages - is key to influencing consumer eye-tracking attention[2]. In recent years, the apparel industry has been the most productive in online marketing, and the most common way apparel brands operate on online platforms is through website layout as well as design of apparel and model images, allowing consumers to rapidly bond with the brand, generate interest and favor, and desire for purchase.

E-commerce marketing information flow includes graphics and text, when unable to touch the actual product, graphic depictions are the best way to raise consumer purchase interests in the virtual internet world. Consumer behavior researcher Elder \& Krishna have noted in their studies: product depictions in advertisements facilitate mental stimulation and evoke heightened consumer purchase intentions[3]. However, consumer decision behavior and perception is a very complicated process[4], consumer purchase intention is affected by the underlying situational context[5]. Most consumers prefer products that are easy to interact with, mainly because of the restrictions of the product's orientation and consumer contextual background that a consumer evaluates how the product interacts, a consumer is dependent upon the setting of which the product is visually depicted: this is seen as an easy way for manipulation and imagination[6]. Through neurological experiments, a study by the psychologists Dijksterhuis \& Arts in 2010 examines and illustrates how consumer attention and goals are unconsciously restricted, and that consumer attention and awareness functions are two separate components within the brain[7].

Using the eye tracker technique to analyze consumer eye movement visual patterns while conducting online shopping behavior, to further examine consumer behavior and decision-making process, and to analyze the stimulus' (i.e. the image, graphic or text shown on the screen) level of attraction, this technique has been widely implemented in marketing, mass communication and other economic and academic related studies. For instance, Wallraven et al. have conducted the eyetracking technique in a study detecting and observing a large number of paintings from different art periods; the study evaluated the participant's visual 
complexity and aesthetic appeal in order to systematically analyze the participant's preference in artistic style[8]. Another experiment that involved eye-tracking examined consumer eye movement trajectory when looking at designer handbags, as well as the duration fixated on a specific region of the product when online shopping, the study analyzed the influencing factors of consumer visual attention [9]. Therefore, we can establish that it is feasible to implement the eye-tracking technique to analyze actual consumer behavior, especially for practical communication on online marketing platforms.

This study focuses on the influence of camera perspectives of online apparel products on product attention and consumer purchase interest. By implementing the eye-tracking experiment, this study provides implications for optimizing visual depiction for online apparel products, intended to assist designers and market practitioners in future strategies for visual website layout and camera perspectives of models and products.

\section{RESEARCH QUESTIONS}

This study intends to examine the relevance between different camera perspectives of online apparel products and consumer attention and purchase interest, the experimental analysis is conducted to observe consumer visual pattern. This study aims to optimize product images for greater attraction and better stimulation for consumer purchase interest. The way products are depicted on websites facilitates mental stimulation and evokes heightened consumer purchase intentions[3], therefore this study proposes the following three research questions:

1. By comparing graphic images to examine apparel product depictions online, including "images with model displaying item" and "enlarged images of single item", and implementing the eye-tracking technique, which kind of image attracts longer visual attention and generates greater interest in the product?

2. On the apparel website, when the image of the product shows a model wearing the item, two kinds of camera perspectives are included: "model's face is visible when displaying the item" and "below head images of the model displaying the item (therefore eliminating the relative factor of the model's face (beautiful or ugly). This is to examine the observer's eye movement.

3. On the apparel website, when the image of the product is shows a model wearing the item, two kinds of depictions are included: "removed backgrounds" and "scenario backgrounds (specific settings)". This is to examine the relationship between the depicted product and the observer's preference and mental stimulation.

\section{METHOD}

\subsection{Participants}

Participants include voluntary 15 female workers, with an average age of 25.7. All participants have at least two years of online apparel shopping experience. The price of purchasing a single apparel ranges between 45 to 60 USD.

\subsection{Stimulus materials}

The author has selected a black dress as this study's observed apparel. The dress is of silk fabric, draping and flowing, slim-looking, and features an elegant taste. For the experiment's stimuli, the images of the dress are shot with a professional model wearing it in a photography studio and in an office scenario. In response to the aforementioned research questions, the stimuli are designed in three categories, each category featuring two images. The first category's question is "Which image do you think is more appropriate to be shown in the main product menu of the online apparel website's main page?" (Fig. 1) The second category's question is "When entering the apparel product's content page, which image depiction do you prefer (model's face visible or model's face not visible)?" (Fig. 2) The third category's question is "Which of the scenario background image or removed background image evoke for you greater imagination and interest for this dress?" (Fig. 3) Each image is defined with a red frame and number within the frame as the regions of interests (ROI) set by the author. These ROI frames are hidden to the participants during the experiment.

\subsection{Instruments and Procedure}

This main instrument implemented in the study's experiment is the Screen Eye Tracker and a laptop computer for showing the visual stimulus materials and tracking eye movement trajectories. The author used the EyeNTNU-180 Screen Eye Tracker, applying infra-red LED to detect eye focus, and 30 $\mathrm{Hz}-180$ sample frequency technique to lower the error rate and increase the experiment's accuracy.

Before the experiment, the participants are given the task of "you are planning to buy a dress that is appropriate for the work environment" and then answer the corresponding questions when comparing the side by side images on the screen. The sample analysis of the eye-tracking data after the experiment includes the following four variants: the number of fixations (NOF), the total contact time (TCT), the latency of first fixation (LFF), the duration of first fixation (DFF). 


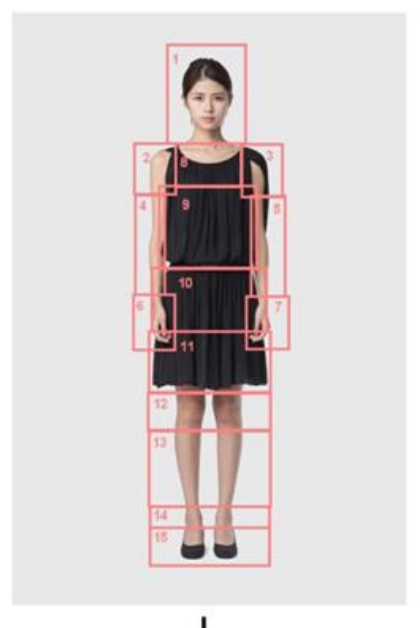

L

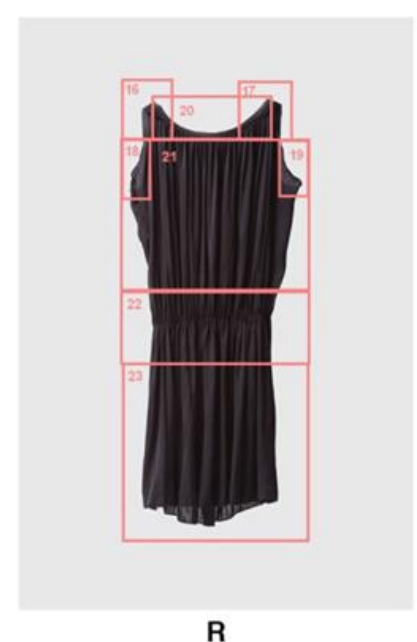

R

Figure 1. L: Model wearing the item vs. R: Enlarged image of the single item

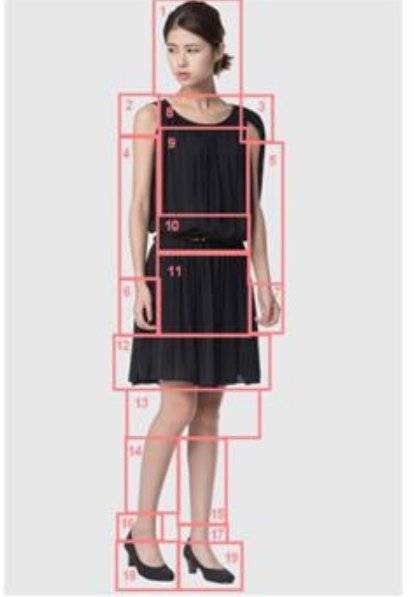

L

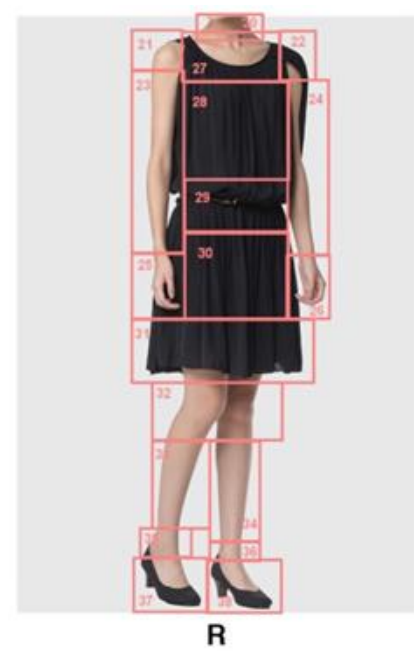

R

Figure 2. L: Model's face visible vs R: Model's face is not visible

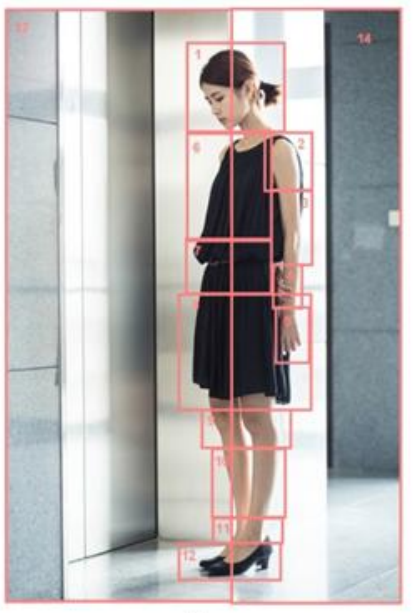

L

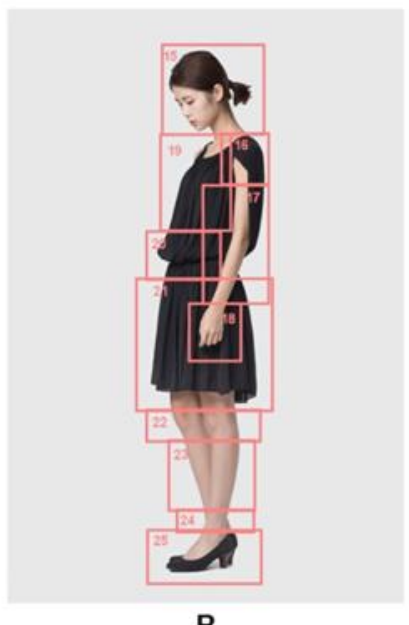

$\mathbf{R}$

Figure 3. L: Scenario background vs. R: Removed background

\section{RESULTS}

\subsection{Data analyses}

The results of the questions from the three categories are used to analyze each group of answers given by the participants, and the four variants mentioned in 3.3 are the main analyses and descriptions to the eyetracking data. First of all, NOF and TCT respectively indicate the number of times and the total duration that visual fixation occurred within the ROI; LFF indicates the type of visual attention participants give to different ROI; and DFF indicates the duration of continuous fixation within the ROI.

\subsection{Results of eye-tracking data: Model displaying item $(L)$ vs. Enlarged image of single item}

$100 \%$ of the participants prefer the apparel image with the model wearing the item. Both image stimuli have a total of 23 ROIs, and the results show only 18 effective fixation data of which $L: R=13: 5$. According to TCT results, the top $6 \mathrm{ROI}$ of both left and right images' data is regionally coded and analyzed, as shown in table 1 , the regions of participants' interests fall accordingly in R1, R9, R21, R8, R10, and R3; which is the model's face, upper image (R9 and R21), neckline, lower image and left shoulder areas.

Table 1. Eye-tracking data for top 6 ROIs. (L representing "model displaying item", $\mathrm{R}$ representing “enlarged image of single item"

\begin{tabular}{|c|c|c|c|c|c|c|}
\hline \multirow{2}{*}{} & \multirow{2}{*}{ ROI } & \multicolumn{2}{|c|}{ NOF } & TCT(ms) & LEF(ms) & DFF(ms) \\
\cline { 3 - 7 } & & Mean & SD & Mean & Mean & Mean \\
\hline L & R1 & 7.82 & 9.90 & 284.55 & 3012.36 & 29.09 \\
\hline R & R21 & 6.91 & 12.42 & 206.09 & 2138.09 & 15.55 \\
\hline L & R9 & 6.82 & 10.87 & 203.55 & 1851.82 & 12.91 \\
\hline L & R8 & 2.55 & 5.96 & 103.09 & 1571.73 & 9.36 \\
\hline L & R10 & 2.36 & 4.86 & 71.45 & 2467.55 & 9.64 \\
\hline L & R3 & 1.64 & 2.29 & 44.55 & 1887.09 & 10.55 \\
\hline
\end{tabular}

\subsection{Results of eye-tracking data: Model's face visible (L) vs Model's face invisible (R)}

$73 \%$ of the participants prefer the product image where the model's face is visible. Both image stimuli have a total of 38 ROIs, and the results show only 27 effective fixation data of which $L: R=15: 12$. According to TCT results, the top 6 ROI of both left and right images' data is regionally coded and analyzed, as shown in table 2 However, 4 ROI falls on where the model's face is not visible (R). The regions of participants' interests fall accordingly in $\mathrm{R} 1, \mathrm{R} 28, \mathrm{R} 24, \mathrm{R} 8, \mathrm{R} 30$, and R20; which is the model's face, upper image, left handcuff, neckline, lower image and neck areas. 
Table 2. Eye-tracking data for top 6 ROIs.(L representing "model's face visible", R representing "model's face invisible"

\begin{tabular}{|c|c|c|c|c|c|c|}
\hline \multirow{2}{*}{} & \multirow{2}{*}{ ROI } & \multicolumn{2}{|c|}{ NOF } & TCT(ms) & LEF(ms) & DFF(ms) \\
\cline { 3 - 7 } & & Mean & SD & Mean & Mean & Mean \\
\hline L & R1 & 4.73 & 6.48 & 154.27 & 1867.45 & 28.73 \\
\hline R & R28 & 4.73 & 7.85 & 140.00 & 3017.18 & 12.18 \\
\hline R & R24 & 2.82 & 3.16 & 111.55 & 3298.91 & 22.18 \\
\hline L & R8 & 2.09 & 5.49 & 96.36 & 653.27 & 4.18 \\
\hline R & R30 & 2.36 & 3.72 & 78.36 & 1978.27 & 17.73 \\
\hline R & R20 & 1.82 & 2.44 & 65.00 & 2780.55 & 22.82 \\
\hline
\end{tabular}

\subsection{Results of eye-tracking data: Scenario background $(L)$ vs Removed background $(R)$}

$82 \%$ of the participants prefer the product image with a scenario background. Both image stimuli have a total of 25 ROIs, and the results show only 20 effective fixation data of which $\mathrm{L}: \mathrm{R}=12: 8$. According to TCT results, the top $6 \mathrm{ROI}$ of both left and right images' data is regionally coded and analyzed, as shown in table 3 . However, 4 ROI falls on where the model's face is not visible $(\mathrm{R})$. The regions of participants' interests fall accordingly in R13, R1, R6, R15, R17, and R14; which presents the right background (elevator), model's face, upper image, left handcuff, and left background area.

Table 3. Eye-tracking data for top 6 ROIs. (L representing "scenario background", R representing "removed background"

\begin{tabular}{|c|c|c|c|c|c|c|}
\hline \multirow{2}{*}{} & \multirow{2}{*}{ ROI } & \multicolumn{2}{|c|}{ NOF } & TCT(ms) & LEF(ms) & DFF(ms) \\
\cline { 3 - 7 } & & Mean & SD & Mean & Mean & Mean \\
\hline L & R13 & 7.00 & 8.92 & 231.18 & 1926.73 & 12.27 \\
\hline L & R1 & 4.36 & 7.43 & 139.09 & 2580.45 & 16.18 \\
\hline L & R6 & 3.18 & 6.63 & 108.91 & 1567.45 & 15.18 \\
\hline R & R15 & 3.09 & 2.74 & 76.00 & 1597.64 & 13.45 \\
\hline R & R17 & 1.36 & 2.62 & 51.55 & 1428.00 & 7.82 \\
\hline L & R14 & 1.73 & 4.24 & 50.27 & 1184.55 & 6.18 \\
\hline
\end{tabular}

\section{CONCLUSION}

E-consumers gain product information by browsing through product images on online websites, the camera perspective of the product images critically influence the level of consumer preference and purchase intention towards the product. The results of the eye-tracking experiment implemented in this study show the consumer preferences for camera perspectives: images with models displaying the item are better than enlarged images of the single item; images with scenario backgrounds receive more visual attention than images with the background removed, images with scenario backgrounds also provide customers greater mental stimulation for use of the product; although the participants answered that they prefer models with their faces visible when displaying the item, however through the eyetracking experiment, results show that images where model's faces are not visible receive greater visual attention.

\section{REFERENCES}

[1] Lewis, D. and Bridger, D. 2002. The Soul of the New Consumer: Authenticity - What We Buy and Why in the New Economy. USA: Nicholas Brealey Publishing.

[2] Song, H. and Schwarz, N. 2008. If it's hard to read, it's hard to do. Processing fluency affects effort prediction and motivation. Psychological Science 19, 986-988.

[3] Elder, R. S., \& Krishna, A. 2012. The "visual depiction effect" in advertising: Facilitating embodied mental simulation through product orientation. Journal of Consumer Research 38(6), 988-1003.

[4] Schwarz, N. 2006. Feelings, fit, and funny effects: A situated cognition perspective. Journal of Marketing Research 43(1), 20-23.

[5] Martino B., Kumaran D., Holt B., Dolan R.J. 2009. The neurobiology of reference-dependent value computation. Journal of Neuroscience, 29(12):3833-42

[6] Eelen, J., Dewitte, S., Warlop, L. 2013. Situated embodied cognition: Monitoring orientation cues affects product evaluation and choice, Journal of Consumer Psychology 23, 424-433.

[7] Dijksterhuis, A. \& Arts, H. 2010. Goals, Attention, and (Un) Consciousness. Annual Review of Psychology, 61, 467490.

[8] Wallraven C., Cunningham D., Rigau J., Feixas M., Sbert M. 2009. Aesthetic appraisal of art - from eye movements to computers. Computational Aesthetics in Graphics, Visualization, and Imaging.

[9] Ho, H.F. 2014. The effects of controlling visual attention to handbags for women in online shops: Evidence from eye movements. Computers in Human Behavior,30,146-152. 